\title{
Experimental Measurement of Nanolayers via Electromagnetic, Near Infrared, and Gamma Radiation
}

\author{
Pavel Fiala ${ }^{1}$, Karel Bartušek ${ }^{2}$, Jarmila Dědková ${ }^{3}$, Radim Kadlec $^{3}$, Přemysl Dohnal ${ }^{3}$

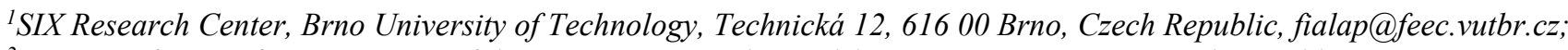 \\ ${ }^{2}$ Institute of Scientific Instruments of the ASCR v.v.i., Kralovopolská, 147, Brno, 612 00, Czech Republic, \\ ${ }^{3}$ Dept. of Theoretical and Experimental Electrical Engineering, Brno University of Technology, Technická 12, 61600 Brno, \\ Czech Republic
}

\begin{abstract}
We discuss and compare the results obtained from experimental measurements of a two-layer, $\mathrm{Ni}$ and $\mathrm{TiO}_{2}$ nanometric structure deposited on siliceous glass. Utilizing previous theoretical models of multilayers or periodic systems and their verifications, the paper focuses on measurement in the NIR, visible, UV, X-ray, and gamma bands of the electromagnetic spectrum; the wavelength of the incident electromagnetic wave is respected. The proposed evaluation comprises a brief description of a Snell's law-based semi-analytic model of electromagnetic wave propagation through a layered material. We also demonstrate the expected anti-reflective and shielding effects in the $\mathrm{X}$-ray and gamma-ray bands, respectively.
\end{abstract}

Keywords: Nanomaterials, multilayered material, resonance, periodic system, electromagnetic wave, X-ray, gamma-ray, antireflection, shielding.

\section{INTRODUCTION}

With the advancement in nanotechnologies, sciences, and engineering [1]-[5], a portion of researchers in theoretical electrical engineering have centered on investigating thin layers [1] and periodic systems [5]. In terms of electromagnetic field characterization, such a focus reflects the transition from macroscopic to microscopic models [13][16], respecting hypotheses on the composition of atomic and subatomic matter [20]-[22]. The basis of this shift lies in the current technological ability to design fundamental electric or electronic components whose dimensions approach those of both the wavelength of the transmitted electromagnetic wave and the basic building blocks of matter, namely, molecules and atoms [5], [7]-[9].

The basic phenomena of nanoelectronics and nanoengineering include, among other effects, the electromagnetic wave and its interaction with an elementary electric charge, resonance in common frequency bands when interacting with investigated objects, and incidence on a layered periodic structure of matter. To date, the theoretical and experimental research into carbon-based single-layer periodic systems has yielded, for example, the relatively recent definition and isolation of graphene, Fig.1. [8], [10], [12]. The mechanical and electrical properties of single-atom layered materials were nevertheless examined already in the mid- $20^{\text {th }}$ century [32], utilizing macroscopic experiments and theoretical assumptions that remained applicable for decades [6], [9], [11], [33], [4]. Another embodiment of the discussed systems, coupled to hydrogen or other atom nuclei, is graphene, Fig.2. [8], [13]. The structures are interesting from the perspective of the transmission of an electromagnetic wave, which can be performed perpendicularly, obliquely, or along the surface of the given structure or layered systems and their combinations. Measurement techniques and relevant evaluations [6], [16]-[17], [19], [26], [30], [31] are presently available to verify the resulting properties of designed nanomaterial architectures; however, the methodical approaches towards measuring the basic electromagnetic features of periodic systems, structures, elements, and particles in the nano-domain have not been appropriately developed to date.

A suitable tool for determining the basic electromagnetic properties of nanostructures consists in low-level, nondestructive methods associated with comparative principles [34]. In procedures where the values of quantities within the continuous frequency spectrum range above units of $\mathrm{nV}$ or $\mathrm{nA}$, the sample being measured is influenced by the actual experimental process, and the obtained data are thus subject to misinterpretation. Such a scenario can be clearly identified in liquid or gaseous (plasmatic) samples [35]-[36]. When used to test solid systems (including, for instance, nanolayers, nanomaterials, or nanoparticles on a solid substrate), the low- 
level approach in general or the related measurement may appear inappropriate; simple experiments nevertheless allow us to find that failing to observe the individual characteristic limits as regards the maximum levels of sensed quantities fundamentally affects the accuracy and credibility of the interpretation of experimentally obtained results.

The article attempts to demonstrate two techniques for examining thin layered materials and offers a perspective of the acquisition of electromagnetic properties of nanomaterials.

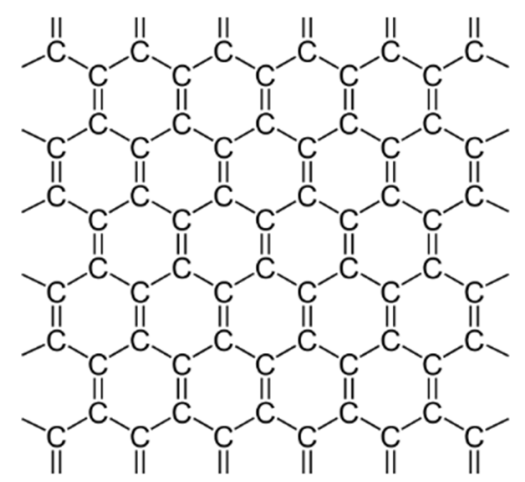

Fig.1. The arrangement of carbon periodic structures: a graphene layer.
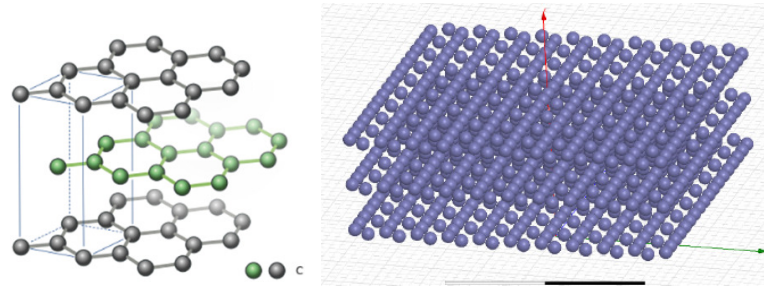

Fig.2. The arrangement of carbon periodic structures: a graphite layer.

Importantly, the applied experiments or analyses have to be built upon specific geometrical and numerical models. This approach then should respect the character of the relevant probabilistic mathematical simulation; the uncertainty of the occurrence of an electric charge $q$; and the frequency of the transmitted signal $f_{\mathrm{t}}$ compared to the first harmonic frequency $f_{\mathrm{a} 1}$ [16]-[19], which is defined by the movement of elementary charged parts of an atom with diameter $d_{\mathrm{a} 1}$. We have

$$
f_{t}<<\frac{c}{d_{a 1}}
$$

where $c$ is the speed of light in vacuum, and $f_{\mathrm{a} 1}=c / d_{\mathrm{a} 1}$. In any geometrical configuration of a periodic nanostructure, it is suitable to analyze or measure the EMG field at the level of structure elements with respect to the evaluation of known macroscopic quantities, including the surface power flux density, electric and magnetic field intensities, and flux densities related to the harmonic behavior of a signal transmitted or reflected on the structure. Then, for the assumed analysis or measurement of an electromagnetic field, we recognize the following subregions:

a) $f_{\mathrm{t}} \in\left\langle 1.10^{6} ; 500.10^{9}\right\rangle[\mathrm{Hz}], f_{\mathrm{a} 1} \approx 2.10^{18}[\mathrm{~Hz}]$, where condition

(1) is satisfied, and

b) $f_{\mathrm{t}} \in\left\langle 1.10^{12} ; \quad 5.10^{19}\right\rangle[\mathrm{Hz}], \quad f_{\mathrm{a} 1} \approx 2.10^{18}[\mathrm{~Hz}]$, where the condition is not satisfied.

In the former domain, the experimentation or measurement are suitably carried out using integration methods within the continuous electromagnetic wave frequency spectrum; in the latter subregion, hybrid or pulse techniques are more appropriate [17], [34].

\section{EXPERIMENTAL MEASUREMENT OF A NANOMETRIC BILAYER}

Fig.3. below displays the tested laboratory sample of two nanolayers deposited on siliceous glass; the dimensions are $70 \times 25 \mathrm{~mm}$, with the thickness of $1 \mathrm{~mm}$. The experiment differs from that presented recently by Vazquez-Velazquez et al. [37]. Each side of the sample is covered with one layer, and the layers form thin materials mutually different from the perspective of the electromagnetic field. The Ni nanolayer is ferromagnetic and has the relative permittivity of $\mu_{\mathrm{r}}>5,000$, whereas the $\mathrm{TiO}_{2}$ layer is dielectric and exhibits the relative permeability of $\varepsilon_{\mathrm{r}}>100$; both of them embody clusters of matter on a glass substrate, which are capable of changing the directions of propagation of the electric and magnetic components of an incident electromagnetic wave. The resulting structure is expected to show a surface effect, namely, the wave propagation directions from the $\mathrm{Ni}$ and $\mathrm{TiO}_{2}$ sides will not be identical.

In the macroscopic interpretation of the electromagnetic field, the layers (having a thickness of approximately $50 \mathrm{~nm}$ ) easily become saturated, and it is then suitable to employ lowlevel measurement for the actual experiment.

The nanolayers were examined in the ultraviolet (No. 1), Xray (No. 2), and gamma ray (No. 3) spectral regions, Fig.4.; the corresponding wavelengths were $\lambda_{\mathrm{UV}}=10-400 \mathrm{~nm}$, $\lambda_{\mathrm{X}}=0.1-10 \mathrm{~nm}$, and $\lambda_{\mathrm{ga}}=1 \mathrm{pm}-0.1 \mathrm{~nm}$, respectively. The measurement devices included a Varian Cary 5E UV-VisNIR spectrophotometer to facilitate the procedures in region No. 1; an EcoRay HF1040 portable X-ray generator (ensuring the exposure voltage of $40-100 \mathrm{kV}$, the X-ray beam current pulse regulation via current $\mathrm{A}-\mathrm{K} \mathrm{d} I_{\text {Xray }}=0.32-50 \mathrm{mAs}$, and the X-ray beam energy of 2-2,500 J, with the corresponding wavelength of $\lambda_{\mathrm{EcR}}=0.49-124 \mathrm{~nm}$ ) to carry out the measurement within region No. 2; and a ${ }^{241} \mathrm{Am}$ weak radiation source completed with a REP 921 Geiger counter for region No. 3, the relevant wavelength being $\lambda_{\mathrm{AM}}=20.8 \mathrm{pm}$. The basic positioning of the devices for the individual measuring cycles is represented in Fig.5. 


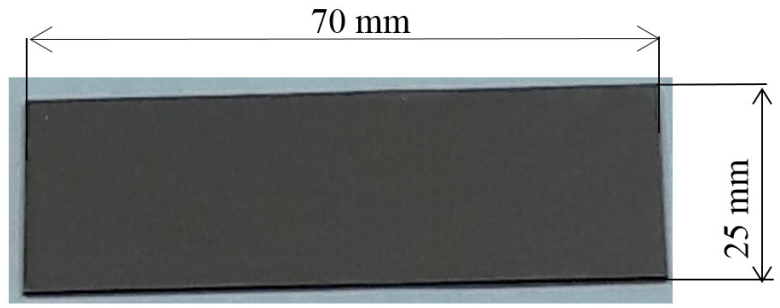

a)

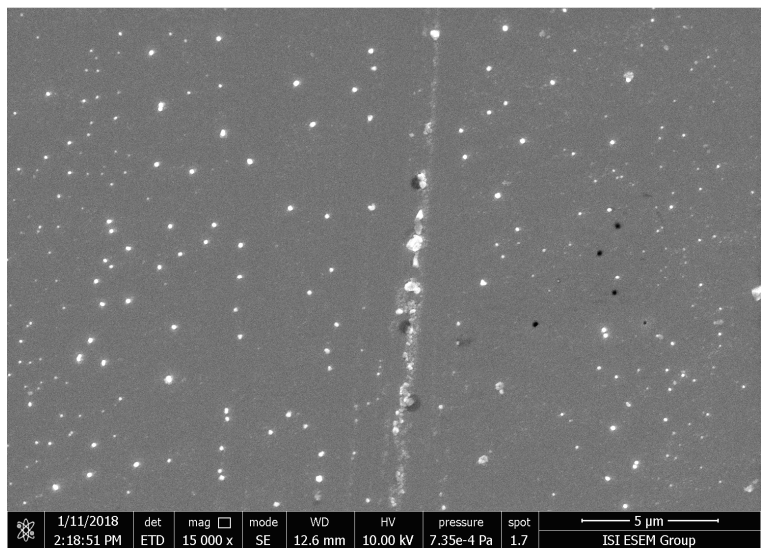

b)

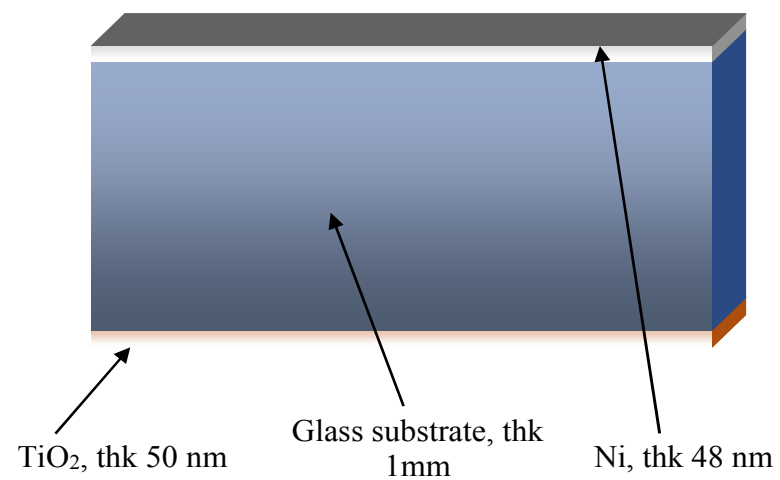

c)

Fig.3. The tested sample: a) the $\mathrm{Ni}$ and $\mathrm{TiO} 2$ nanolayers (48 $\mathrm{nm}$ and $50 \mathrm{~nm}$ in thickness, respectively) on siliceous glass ( $\mathrm{SiO} 2)$ having the thickness of $1 \mathrm{~mm}$; b) the surface on the Ni side; c) a schematic drawing of the deposited layers.

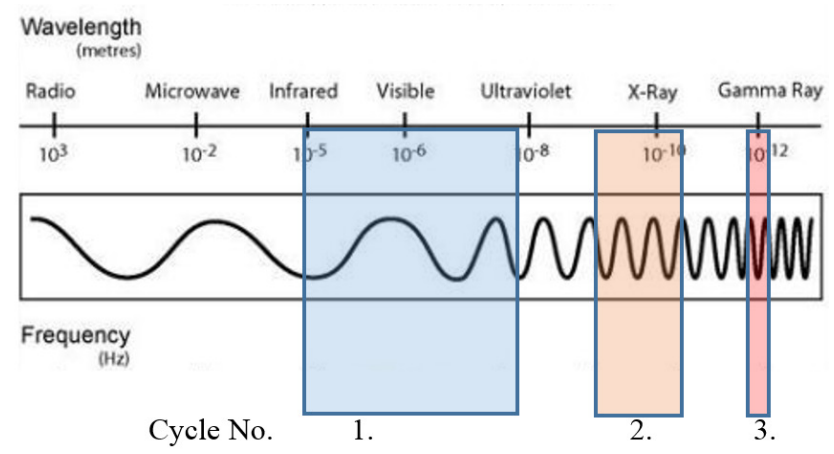

Fig.4. The regions of the electromagnetic spectrum utilized in testing the $\mathrm{Ni}(48 \mathrm{~nm})$ a $\mathrm{TiO} 2(50 \mathrm{~nm})$ nanolayers deposited on siliceous glass.

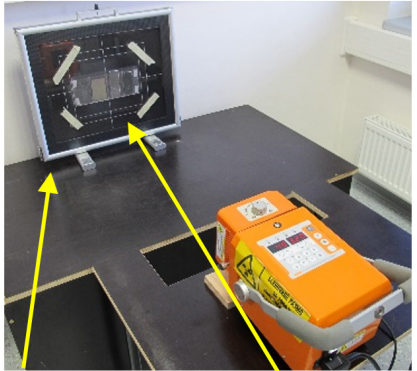

X-ray digital detector samples of the material X-ray source

a)

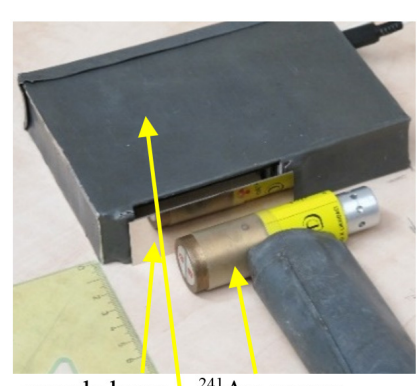

sample layer $\quad{ }^{241} \mathrm{Am}$ source Geiger counter
Fig.5. The setup to enable testing within the ultraviolet, a) X-ray, and b) gamma ray regions.

\section{EVALUATION OF THE EXPERIMENT UV - X-RAY}

The layers were measured at various laboratories, prominently including those managed by the Brno-based Institute of Scientific Instruments, where the ultraviolet cycles were carried out. In these procedures, under UV radiation in the wavelength range of $\lambda_{\mathrm{UV}}=190 \mathrm{~nm}-1,100 \mathrm{~nm}$, the sample bilayer exhibited the attenuation values between 80 and $90 \mathrm{~dB}$; the layers can therefore be characterized as attenuating for the given wavelength range.

When performing the X-ray tests, using also $\mathrm{Al}, \mathrm{Pb}$, and $\mathrm{Fe}$ tablets as reference samples whose behavior under exposure to X-ray or gamma radiation is predictable, we progressively set the parameters of the generator and arranged the samples such that the $\mathrm{Ni}$ and $\mathrm{TiO}_{2}$ layers deposited on two separate $\mathrm{SiO}_{2}$ glass substrates could face the incident electromagnetic wave simultaneously (Fig.6.).

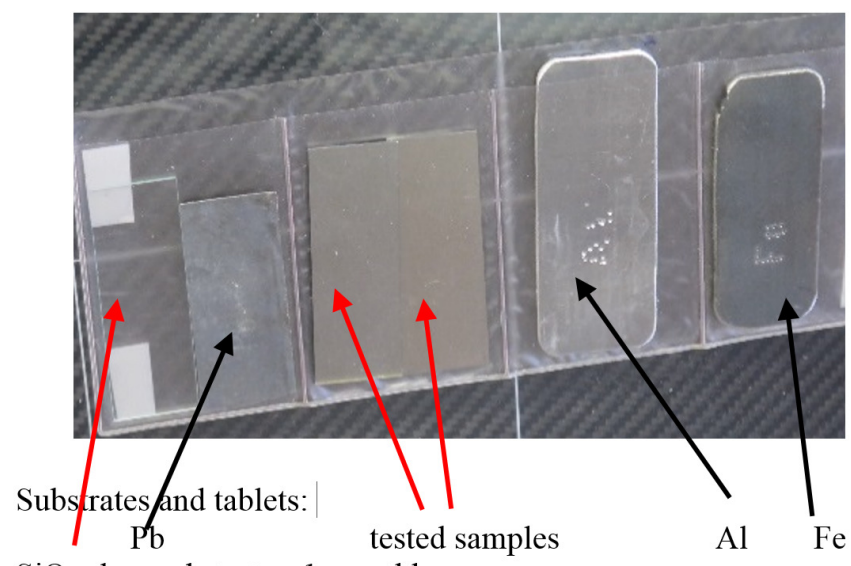

$\mathrm{SiO}_{2}$ glass substrates, $1 \mathrm{~mm}$ thk

Fig.6. The samples configured for the $\mathrm{X}$-ray testing of the $\mathrm{Ni}$ $(48 \mathrm{~nm})$ and $\mathrm{TiO}_{2}(50 \mathrm{~nm})$ layers deposited on siliceous glass.

The anode-cathode voltage provided by the X-ray source varied between 40 and $100 \mathrm{kV}$, and the current pulse length was changed gradually; thus, the energy of the X-ray radiation generating beam was altered parametrically, with the assumed wavelength of $\lambda_{\text {Xray }}=10 \mathrm{~nm}-0.1 \mathrm{~nm}$. The results of the experiment and the related evaluation are shown in Table 1. and Fig.7., respectively. 
Table 1. The parametric setting of the X-ray generator and evaluation of the absorptivity (red color) and reflectivity (black color) of the $\mathrm{Ni}$ and $\mathrm{TiO}_{2}$ bilayer.

\begin{tabular}{|l|l|l|l|}
\hline Num. & $\boldsymbol{U}_{\mathrm{A}-\mathbf{K}}[\mathbf{k V}]$ & $\mathbf{d} \boldsymbol{I} . \boldsymbol{t}[\mathbf{A s}]$ & $\boldsymbol{W}[\mathbf{J}]$ \\
\hline$\underline{1}$ & 40 & $3.2 \cdot 10^{-4}$ & 12.8 \\
\hline$\underline{2}$ & 40 & $10 \cdot 10^{-4}$ & 40 \\
\hline 3 & 40 & $50 \cdot 10^{-4}$ & 200 \\
\hline 4 & 40 & $100 \cdot 10^{-4}$ & 400 \\
\hline 5 & 40 & $160 \cdot 10^{-4}$ & 640 \\
\hline 6 & 40 & $400 \cdot 10^{-4}$ & 1600 \\
\hline 7 & 40 & $800 \cdot 10^{-4}$ & 3200 \\
\hline 8 & 40 & $1000 \cdot 10^{-4}$ & 4000 \\
\hline 9 & 40 & $500 \cdot 10^{-4}$ & 2000 \\
\hline 10 & 40 & $630 \cdot 10^{-4}$ & 2520 \\
\hline 1 & 50 & $100 \cdot 10^{-4}$ & 500 \\
\hline 2 & 50 & $160 \cdot 10^{-4}$ & 800 \\
\hline 3 & 50 & $120 \cdot 10^{-4}$ & 620 \\
\hline 1 & 60 & $10 \cdot 10^{-4}$ & 60 \\
\hline 2 & 60 & $50 \cdot 10^{-4}$ & 300 \\
\hline 3 & 60 & $100 \cdot 10^{-4}$ & 600 \\
\hline 1 & 70 & $10 \cdot 10^{-4}$ & 70 \\
\hline 2 & 70 & $50 \cdot 10^{-4}$ & 350 \\
\hline 3 & 70 & $63 \cdot 10^{-4}$ & 441 \\
\hline
\end{tabular}

\begin{tabular}{|l|l|l|l|}
\hline Num. & $\boldsymbol{U}_{\mathbf{A}-\mathbf{K}}[\mathbf{k V}]$ & $\mathbf{d} \boldsymbol{I} \cdot \boldsymbol{t}[\mathbf{A s}]$ & $\boldsymbol{W}[\mathbf{J}]$ \\
\hline 1 & 80 & $10 \cdot 10^{-4}$ & 500 \\
\hline 2 & 80 & $50 \cdot 10^{-4}$ & 800 \\
\hline 3 & 80 & $40 \cdot 10^{-4}$ & 620 \\
\hline 4 & 80 & $32 \cdot 10^{-4}$ & 60 \\
\hline 5 & 80 & $25 \cdot 10^{-4}$ & 300 \\
\hline 1 & 90 & $10 \cdot 10^{-4}$ & 90 \\
\hline 2 & 90 & $40 \cdot 10^{-4}$ & 360 \\
\hline 3 & 90 & $32 \cdot 10^{-4}$ & 288 \\
\hline 4 & 90 & $25 \cdot 10^{-4}$ & 225 \\
\hline 5 & 90 & $20 \cdot 10^{-4}$ & 180 \\
\hline 1 & 100 & $10 \cdot 10^{-4}$ & 100 \\
\hline 2 & 100 & $13 \cdot 10^{-4}$ & 125 \\
\hline 3 & 100 & $20 \cdot 10^{-4}$ & 200 \\
\hline
\end{tabular}

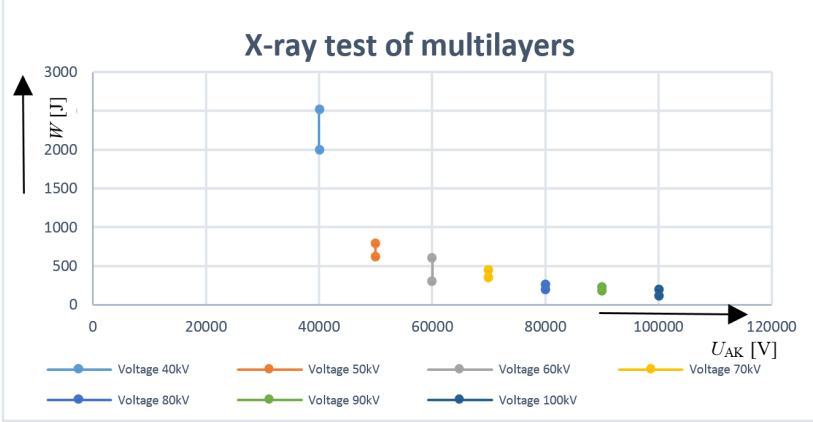

Fig.7. The monitored antireflective effect in the $\mathrm{Ni}$ and $\mathrm{TiO}_{2}$ layers related to the preset voltages.

The experiment demonstrated that the bilayer exhibits antireflective properties at certain X-ray beam energy settings. In this context, Fig.8. details the exposures for both the setting at which the electromagnetic wave is damped by the samples
(Fig.8.a)) and the configuration that yields the anti-reflective effect (Fig.8.b)). As is obvious from Fig.8.b), the bilayer samples on the glass substrates are transparent, while the substrate alone displays wave attenuation with respect to its environment.

\section{EVALUATION OF THE EXPERIMENT GAMMA-RAY}

Within the gamma-ray region, we carried out 39 repeated measurement cycles using the Geiger counter (Fig.5.b)); the electromagnetic radiation was produced by a ${ }^{241} \mathrm{Am}$ source having the wavelength of $\lambda_{\mathrm{Am}}=20.8 \mathrm{pm}$.

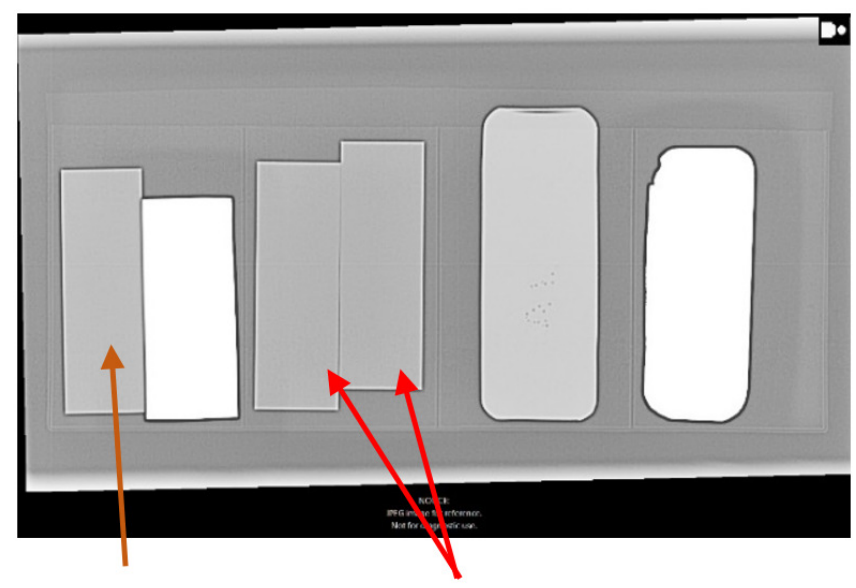

a) substrate tested samples

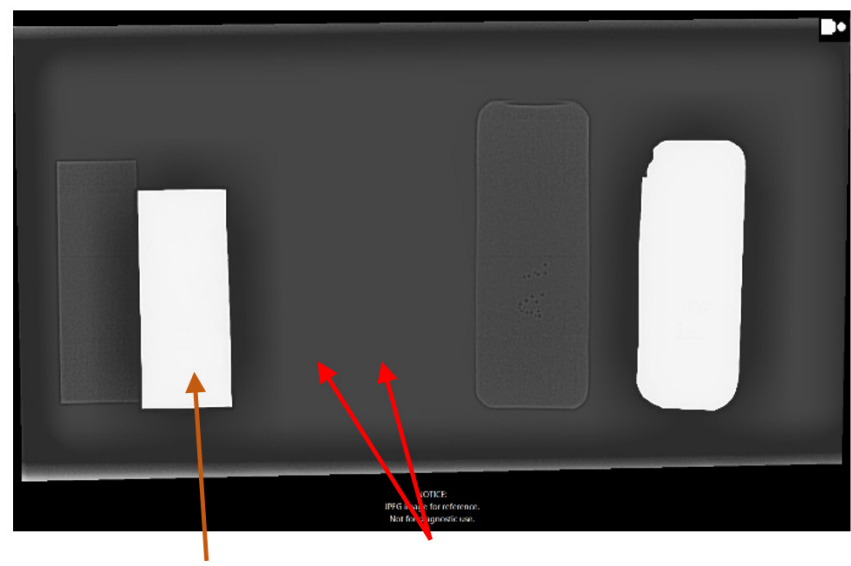

b) substrate tested samples

Fig.8. The attenuation of the samples in the X-ray nanolayer testing procedure: a) all examined items; b) transparency properties and conditions (the anti-reflective effect).

We evaluated the natural background and direct radiation, focusing on the number of pulses without the samples, with the substrate, and with the nanolayers positioned towards the incident electromagnetic wave. The experimental results, namely, data concerning the number of pulses per 100 seconds and the standard deviation, are summarized in Table 2. and Fig.9.

The evaluated incidence of an electromagnetic wave emitted by the ${ }^{241} \mathrm{Am}$ source indicates that each of the layers has its specific transparency properties. 
Table 2. The attenuation of the nanolayer samples achieved using the ${ }^{241} \mathrm{Am}$ source.

\begin{tabular}{|l|l|l|l|l|l|}
\hline SOURCE & NONE & ${ }^{241} \mathbf{A M}$ & ${ }^{241} \mathbf{A M}$ & ${ }^{241} \mathbf{A M}$ & ${ }^{241} \mathbf{A M}$ \\
\hline $\begin{array}{l}\text { TYPE OF } \\
\text { MEASUREM } \\
\text { ENT }\end{array}$ & $\begin{array}{l}\text { Background } \\
\text { no sample }\end{array}$ & No sample & Substrate & $\mathrm{Ni}^{-\mathrm{TiO}_{2}}$ & $\mathrm{TiO}_{2}-\mathrm{Ni}$ \\
\hline $\begin{array}{l}\text { AVERAGE } \\
\text { NUMBER OF } \\
\text { PULSES [-] }\end{array}$ & 30.34 & 138.55 & 139.34 & 111.63 & 145.18 \\
\hline $\begin{array}{l}\text { STANDARD } \\
\text { DEVIATION } \\
\text { [-] }\end{array}$ & 6.06 & 11.59 & 9.57 & 12.03 & 14.89 \\
\hline CYCLES & 39 & 39 & 39 & 39 & 39 \\
\hline
\end{tabular}

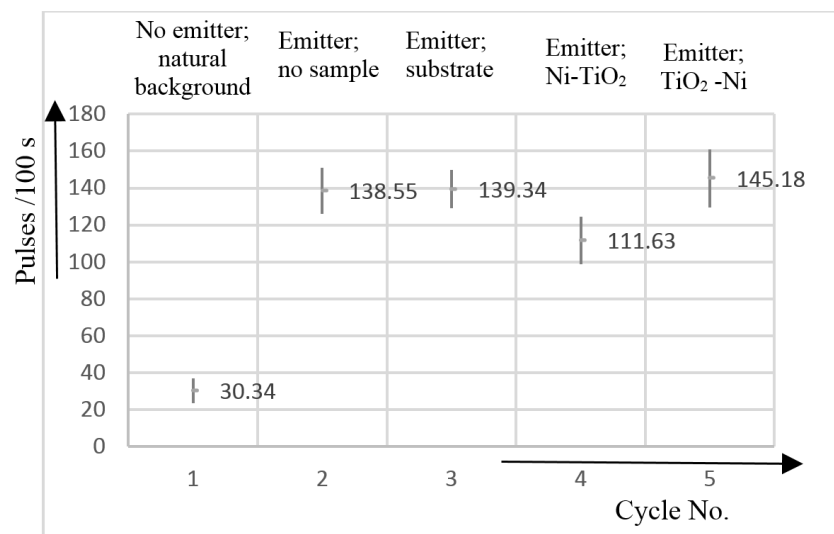

Fig.9. The attenuation of the samples in the X-ray nanolayer testing procedure: a) all examined items; b) transparency properties and conditions (the anti-reflective effect).

\section{NUMERICAL MODEL OF THE MULTILAYER SAMPLES}

Applying Snell's law [23], [29], we obtain the formula to evaluate, from the macroscopic perspective, the wave refraction angle on the discontinuous boundary of two electromagnetically different media [25]. We then have

$$
\frac{\sin \theta_{0}}{\sin \boldsymbol{\theta}_{2}}=\frac{\mathbf{k}_{2}}{\mathbf{k}_{1}}
$$

where $\mathbf{k}$ denotes the wave number with electromagnetic (EMG) wave propagation data, formulated as

$$
\mathbf{k}=\sqrt{-\mathrm{j} \omega \mu \cdot(\gamma+\mathrm{j} \omega \varepsilon)}
$$

where $\varepsilon$ is the permittivity, $\mu$ the permeability, and $\gamma$ the conductivity of the medium. The macroscopic characteristics describing the electromagnetic properties of the materials generally range within the complex domain and markedly depend on the frequency of the incident electromagnetic wave. For simplification, in frequency regions with $f>1 \mathrm{THz}$ we often assume $\mu_{\mathrm{r}}=1$.

Generalized Snell's law (2) can be expressed in components [15]-[16]. The real component of the wave number $k^{\prime} \mathbf{u}_{\mathrm{n} i}^{\prime}$ forms the angle $\varphi$ with the normal vector $\mathbf{u}_{\mathrm{n}}$, and the imaginary component of the wave number $k^{\prime \prime} \mathbf{u}_{\mathrm{n} i}^{\prime \prime}$ makes the angle $v$ with the same vector $\mathbf{u}_{\mathrm{n}}$. If the boundary conditions and generalized Snell's law (2) are valid, we also have $\mathbf{k} \mathbf{u}_{\mathrm{n} i}=\mathrm{k}^{\prime}\left(\mathbf{u}_{\mathrm{n}} \cdot \cos \varphi+\mathbf{t} \cdot \sin \varphi\right)+\mathrm{ik} \mathrm{k}^{\prime \prime}\left(\mathbf{u}_{\mathrm{n}} \cdot \cos v+\mathbf{t} \cdot \sin v\right)$.

After modification [15]-[16], we obtain the formulas and the itemized Snell's law in real variables is expressed as

$$
\begin{aligned}
& k_{1}^{\prime} \sin \varphi_{1}=k_{2}^{\prime} \sin \varphi_{2}, \\
& k_{1}^{\prime \prime} \sin v_{1}=k_{2}^{\prime \prime} \sin v_{2},
\end{aligned}
$$

where $k_{2}^{\prime}$ is the real component, forming the angle $\varphi_{2}$ with the perpendicular line at the boundary, and $k_{2}^{\prime \prime}$ is the imaginary component, making the angle $v_{2}$ with the perpendicular line at the boundary, the $v=0$ or $v=\pi$, as is obvious from Fig.11.b).

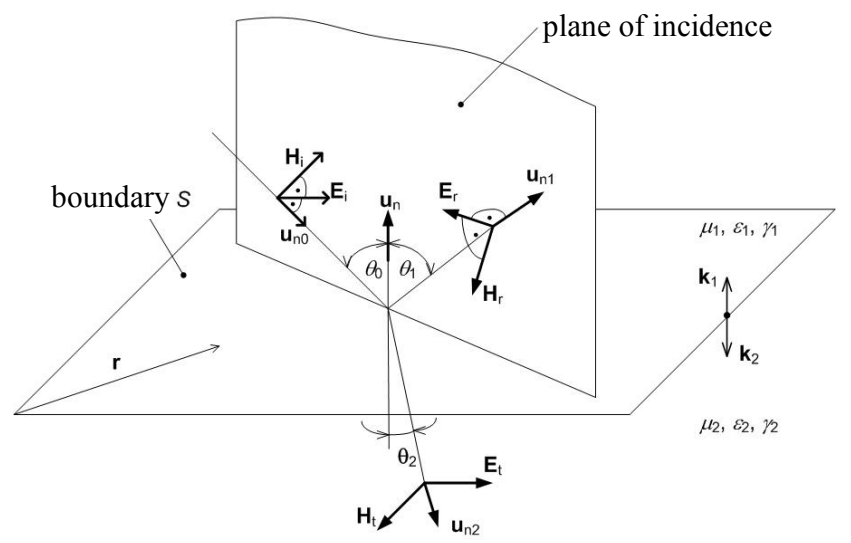

Fig.10. The reflection and refraction of a planar electromagnetic wave: a beam model.

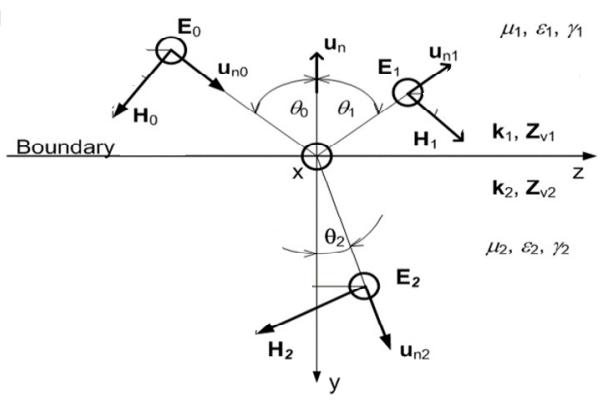

a)

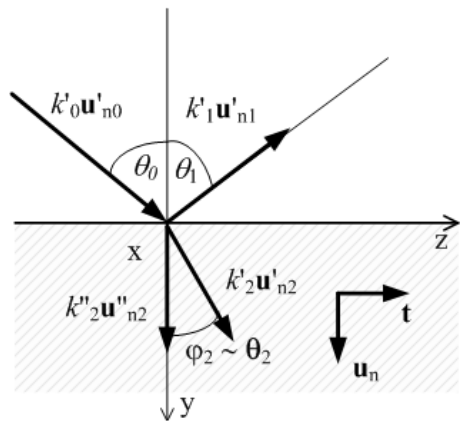

b)

Fig.11. a) The reflection and refraction of an EMG TE wave, [23]; b) the transmission of the constant phase and amplitude. 
An electromagnetic wave is understood as the electric $\mathbf{E}$ and magnetic field $\mathbf{H}$ intensities. The electric E, $\mathbf{D}$ and magnetic H, B components of the incident TE EMG wave according to Fig.11.a) follow from the formula below, [15]-[16], [23], [25]:

$$
\mathbf{E}_{\mathrm{i}}=\mathbf{E}_{0} \mathrm{e}^{-\mathrm{j} \mathbf{k}_{1} \mathbf{u}_{\mathrm{n} 0} \cdot \mathbf{r}}, \mathbf{H}_{\mathrm{i}}=\frac{\mathbf{u}_{\mathrm{n} 0} \times \mathbf{E}_{\mathrm{i}}}{\mathbf{Z}_{\mathrm{v} 1}},
$$

where $\mathbf{E}_{\mathbf{0}}$ is the amplitude of the electric field intensity on the boundary line, $\mathbf{r}$ is the positional vector, and $\mathbf{u}_{\mathbf{n} 0}$ denotes the unit vector of the propagation direction, $\mathbf{Z}_{\mathrm{v} 1}$ is the wave impedance. The model analyzes separately the $\mathbf{E}$ vector parallel to the boundary (the TE EMG wave) as indicated in Fig.11.a) and also the $\mathbf{H}$ magnetic field intensity vector parallel to the interface (the TM EMG wave). For the TE EMG wave, the electric field intensity $\mathbf{E}_{\mathrm{r}}$ of the reflection and $\mathbf{E}_{\mathrm{t}}$ of the transmission beams is written as

$$
\begin{aligned}
& \mathbf{E}_{\mathrm{r}}=\mathbf{E}_{1} \mathrm{e}^{-\mathrm{j} \mathbf{k}_{1} \mathbf{u}_{\mathrm{n} 1} \times \mathbf{r}}, \quad \mathbf{E}_{\mathrm{t}}=\mathbf{E}_{2} \mathrm{e}^{-\mathrm{j} \mathbf{k}_{2} \mathbf{u}_{\mathrm{n} 2} \times \mathbf{r}}, \\
& \mathbf{E}_{\mathrm{r}}=\frac{\mu_{2} \mathbf{k}_{1} \cos \theta_{0}-\mu_{1} \sqrt{\mathbf{k}_{2}^{2}-\mathbf{k}_{1}^{2} \sin ^{2} \theta_{0}}}{\mu_{2} \mathbf{k}_{1} \cos \theta_{0}+\mu_{1} \sqrt{\mathbf{k}_{2}^{2}-\mathbf{k}_{1}^{2} \sin ^{2} \theta_{0}}} \mathbf{E}_{0} \cdot \mathrm{e}^{-\mathrm{j} \mathbf{k}_{1} \mathbf{u}_{\mathrm{n} 1} \times \mathbf{r}}, \\
& \mathbf{E}_{\mathrm{t}}=\frac{2 \mu_{2} \mathbf{k}_{1} \cos \theta_{0}}{\mu_{2} \mathbf{k}_{1} \cos \theta_{0}+\mu_{1} \sqrt{\mathbf{k}_{2}^{2}-\mathbf{k}_{1}^{2} \sin ^{2} \theta_{0}}} \mathbf{E}_{0} \cdot \mathrm{e}^{-\mathrm{j} \mathbf{k}_{2} \mathbf{u}_{\mathrm{n} 2} \times \mathbf{r}} .
\end{aligned}
$$

where $\mathbf{E}_{\mathbf{1}}$ is evaluated from the intensity on the boundary $\mathbf{E}_{\mathbf{0}}$ and the reflection coefficient $\rho_{\mathrm{E}}$, and $\mathbf{E}_{2}$ is obtained from the intensity on the boundary $\mathbf{E}_{\mathbf{0}}$ and the transmission factor $\tau_{\mathrm{E}}$ :

$$
\mathbf{E}_{1}=\rho_{\mathrm{E}} \cdot \mathbf{E}_{0}, \quad \mathbf{E}_{2}=\tau_{\mathrm{E}} \cdot \mathbf{E}_{0}
$$

The magnetic components $\mathbf{H}_{0}, \mathbf{H}_{1}, \mathbf{H}_{2}$ are acquired from the components of the electrical intensities $\mathbf{E}_{0}, \mathbf{E}_{1}, \mathbf{E}_{2}$ and wave impedances $\mathbf{Z}_{\mathrm{v} 1}, \mathbf{Z}_{\mathrm{v} 2}$ :

$$
\mathbf{H}_{0}=\frac{\mathbf{E}_{0}}{\mathbf{Z}_{\mathrm{v} 1}}, \mathbf{H}_{1}=\frac{\mathbf{E}_{1}}{\mathbf{Z}_{\mathrm{v} 1}}, \mathbf{H}_{2}=\frac{\mathbf{E}_{2}}{\mathbf{Z}_{\mathrm{v} 2}}
$$

The reflection coefficient $\rho_{\mathrm{E}}$ and the transmission factor $\tau_{\mathrm{E}}$ via utilizing the wave impedance $\mathbf{Z}_{\mathrm{v} 1}, \mathbf{Z}_{\mathrm{v} 2}$, are evaluated as follows:

$$
\begin{gathered}
\rho_{\mathrm{E}}=\frac{\mathbf{E}_{1}}{\mathbf{E}_{0}}=\frac{\mathbf{Z}_{\mathrm{v} 2} \cos \theta_{1}-\mathbf{Z}_{\mathrm{v} 1} \cos \boldsymbol{\theta}_{2}}{\mathbf{Z}_{\mathrm{v} 2} \cos \theta_{1}+\mathbf{Z}_{\mathrm{v} 1} \cos \theta_{2}} \\
\tau_{\mathrm{E}}=\frac{\mathbf{E}_{2}}{\mathbf{E}_{0}}=\frac{2 \mathbf{Z}_{\mathrm{v} 2} \cos \theta_{1}}{\mathbf{Z}_{\mathrm{v} 2} \cos \theta_{1}+\mathbf{Z}_{\mathrm{v} 1} \cos \theta_{2}} .
\end{gathered}
$$

The formulas of the magnetic component EMG wave are written as

$$
\begin{aligned}
& \mathbf{H}_{\mathrm{r}}=\frac{\mathbf{u}_{\mathrm{n} 1} \times \mathbf{E}_{\mathrm{r}}}{\mathbf{Z}_{\mathrm{v} 1}}, \mathbf{H}_{\mathrm{t}}=\frac{\mathbf{u}_{\mathrm{n} 2} \times \mathbf{E}_{\mathrm{t}}}{\mathbf{Z}_{\mathrm{v} 2}} \\
& \mathbf{H}_{\mathrm{r}}=-\frac{\frac{\mu_{2}}{\mu_{1}} \mathbf{k}_{1} \cos \theta_{0}-\sqrt{\mathbf{k}_{2}^{2}-\mathbf{k}_{1}^{2} \sin ^{2} \theta_{0}}}{\mu_{2} \cos \theta_{0}+\frac{\mu_{1}}{\mathbf{k}_{1}} \sqrt{\mathbf{k}_{2}^{2}-\mathbf{k}_{1}^{2} \sin ^{2} \theta_{0}}} \frac{\mathbf{E}_{0}}{\omega} \cdot \mathrm{e}^{-\mathrm{j} \mathbf{k}_{1} \mathbf{u}_{\mathrm{n} 1} \cdot \mathbf{r}}, \\
& \mathbf{H}_{\mathrm{t}}=-\frac{2 \mathbf{k}_{2} \cos \theta_{0}}{\mu_{2} \cos \theta_{0}+\frac{\mu_{1}}{\mathbf{k}_{1}} \sqrt{\mathbf{k}_{2}^{2}-\mathbf{k}_{1}^{2} \sin ^{2} \theta_{0}}} \frac{\mathbf{E}_{0}}{\omega} \cdot \mathrm{e}^{-\mathrm{j} \mathbf{k}_{2} \mathbf{u}_{\mathrm{n} 2} \cdot \mathbf{r}} .
\end{aligned}
$$

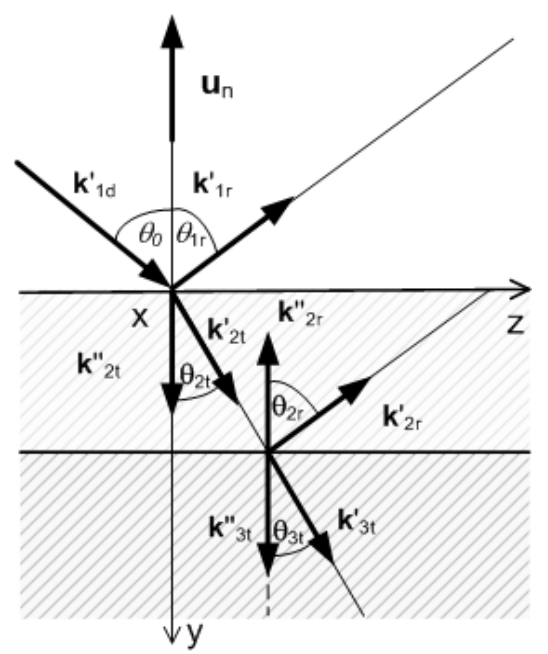

Fig.12. The distribution of the electromagnetic wave components, wave number, and components depending on the propagation direction; the expression of the constant phase and amplitude.

The propagation of an electromagnetic wave on the two layers (Fig.11.) then embodies an analogy of the process on one layer, as outlined in the above formulas $(8-12)$ to express the incident, reflected, and permeating electric and magnetic components of an electromagnetic wave.

For a multi-layered medium [15]-[16], was derived the algorithm [29]. The reflection of the electric component $\mathbf{E}_{\mathrm{r} 0}$, $\mathbf{E}_{\mathrm{t} 0}$ of the EMG field intensity on the first layer is described as

$$
\begin{aligned}
& \mathbf{E}_{\mathrm{r} 0}=\mathbf{E}_{\mathrm{i} 0} \rho_{\mathrm{E} 0} \cdot \mathrm{e}^{-\mathrm{j} \mathbf{k}_{1} \mathbf{u}_{\mathrm{nr} 0} \times \mathbf{r}_{0}}, \\
& \mathbf{E}_{\mathrm{t} 0}=\mathbf{E}_{\mathrm{i} 0} \tau_{\mathrm{E} 0} \cdot \mathrm{e}^{-\mathrm{j} \mathbf{k}_{2} \mathbf{u}_{\mathrm{nt} 0} \times \mathbf{r}_{0}} .
\end{aligned}
$$

The reflection and refraction of the electric component $\mathbf{E}_{\mathrm{r}}$, $\mathbf{E}_{\mathrm{t}}$ of the intensity EMG wave on the following layer is expressed as

$$
\mathbf{E}_{\mathrm{r} l}=\mathbf{E}_{\mathrm{i} l} \rho_{\mathrm{E} l} \cdot \mathrm{e}^{-\mathrm{j} \mathbf{k}_{(l+1)} \mathbf{u}_{\mathrm{nr} l} \times \mathbf{r}_{l}}
$$




$$
\mathbf{E}_{\mathrm{t} l}=\mathbf{E}_{\mathrm{i} l} \tau_{\mathrm{E} l} \cdot \mathrm{e}^{-\mathrm{j} \mathbf{k}_{(l+1)} \mathbf{u}_{\mathrm{nt} l} \times \mathbf{r}_{l}},
$$

where $\mathbf{E}_{\mathrm{r} l}$ a $\mathbf{E}_{\mathrm{t} l}$ are the reflection and refraction electric intensities of EMG waves on the boundary line $l(l=1, \ldots$, max) according to Fig.12., $\mathbf{E}_{\mathrm{i} /}$ denotes the maximum value of the electric field intensity on the boundary line $l$. The magnetic components $\mathbf{H}_{\mathrm{r} 0}, \mathbf{H}_{\mathrm{t} 0}$ of the EMG field intensity for the reflection on the first layer are

$$
\begin{aligned}
& \mathbf{H}_{\mathrm{r} 0}=-\mathbf{H}_{\mathrm{i} 0} \rho_{\mathrm{H} 0} \cdot \mathrm{e}^{-\mathrm{j} \mathbf{k}_{1} \mathbf{u}_{\mathrm{n} 0} \times \mathbf{r}_{0},}, \\
& \mathbf{H}_{\mathrm{t} 0}=-\mathbf{H}_{\mathrm{i} 0} \tau_{\mathrm{H} 0} \cdot \mathrm{e}^{-\mathrm{j} \mathbf{k}_{2} \mathbf{u}_{\mathrm{n} 0} \times \mathbf{r}_{0}},
\end{aligned}
$$

the reflection and transmission $\mathbf{H}_{\mathrm{rl}}, \mathbf{H}_{\mathrm{tl}}$ of the magnetic field intensity on next layers is written as

$$
\begin{array}{r}
\mathbf{H}_{\mathrm{r} l}=-\mathbf{H}_{\mathrm{i} l} \rho_{\mathrm{H} l} \cdot \mathrm{e}^{-\mathrm{j} \mathbf{k}_{(l+1)} \mathbf{u}_{\mathrm{nrl}} \times \mathbf{r}_{l}}, \\
\mathbf{H}_{\mathrm{t} l}=-\mathbf{H}_{\mathrm{i} l} \tau_{\mathrm{H} l} \cdot \mathrm{e}^{-\mathrm{j} \mathbf{k}_{(l+2)} \mathbf{u}_{\mathrm{ntl}} \times \mathbf{r}_{l},},
\end{array}
$$

where $\mathbf{H}_{\mathrm{r} l}$ and $\mathbf{H}_{\mathrm{t} l}$ are the reflection and transmission magnetic components at the boundary $l$.

The above-presented numerical model (7-16) of the reflection and refraction of EMG waves [26] is applicable in an effective analysis utilizing the macroscopic interpretation of the discussed problem. The model allows us to evaluate in a transparent manner the behavior of the layers (which are homogeneous at the macroscopic scale, (1), condition A)) and to set up the experiment. A corresponding analysis according to (1), B) can be suitably performed with models respecting the macroscopic perspective, such as those that exploit the FEM [27]-[28] or the nanoscopic approach [20]-[22]. In the experiment, we assumed the model based on the macroscopic view of electromagnetic wave reflection as defined by Snell's law (7-16).

\section{RESULTS OF THE EXPERIMENTAL MEASUREMENT}

In the designed test of the $\mathrm{Ni}$ and $\mathrm{TiO}_{2}$ bilayers deposited on a siliceous glass substrate having the above-defined thicknesses and exhibiting high relative permeability and permittivity $\mu_{\mathrm{r}}$ and $\varepsilon_{\mathrm{r}}$, respectively, the obtained data can be evaluated [23]-[24] based on the executed experiments for the wavelengths $\lambda_{\mathrm{UV}}=190 \mathrm{~nm}-1,100 \mathrm{~nm}, \lambda_{\mathrm{X} \text {-ray }}=10 \mathrm{~nm}-0.1 \mathrm{~nm}$, $\lambda_{\mathrm{Am}}=20.8 \mathrm{pm}$.

At the $\lambda_{\mathrm{UV}}$ wavelengths in domain A, formula (1), attenuation was observed when the wavelength $\lambda_{\mathrm{UV}}$ of the electromagnetic radiation source had exceeded the thicknesses, namely, $\mathrm{Th}_{\mathrm{Ni}}=48 \mathrm{~nm}$ and $\mathrm{Th}_{\mathrm{TiO} 2}=50 \mathrm{~nm}$; structurally, the layers appear to be macroscopic, and according to the formulas related to reflection and transmission in multilayered materials (11) (13-16), the reflection occurs due to macroscopic properties of the layers (the $\mathrm{Ni}$ layer is ferromagnetic and the $\mathrm{TiO}_{2}$ one dielectric, with the relative permittivities of $\mu_{\mathrm{r}}>5,000$ and $\mathcal{E}_{\mathrm{r}}>100$, respectively).

At $\lambda_{\mathrm{X} \text {-ray }}$, the wavelengths range between domains $\mathrm{A}$ and $\mathrm{B}$, (1). The relevant measurement showed that, under certain conditions (the preset anode-cathode voltage or current pulse), the perpendicular incidence of an electromagnetic wave was accompanied by a state in which a deposited bilayer appeared as anti-reflective for X-ray beams. Based on the formulas defining the reflection and transmission of a wave onto a multilayered material (7-16), the deposited layers cannot be strictly considered macroscopic (continuous); simultaneously, it is necessary to respect the length of the incident electromagnetic wave, which determines the applicable approach from within the macro-, micro-, or nanoscopic set of options usually employed in periodic structures, such as those examined herein [15]-[18]. Assuming suitable incidence of an electromagnetic wave, the structures may provide conditions for improved electromagnetic impedance matching and the resulting antireflective effect.

At $\lambda_{\mathrm{Am}}$, the wavelength is within domain $\mathrm{B},(1)$. The dedicated measurement indicated that, in this band, there already occur effects common for periodic structures [17][18], [27], in accordance with the adopted approach to analyzing the examined problem.

\section{CONCLUSIONS}

Considering the wavelength of an electromagnetic wave incident upon the $\mathrm{Ni}$ and $\mathrm{TiO}_{2}$ sample nanolayers deposited on siliceous glass, we performed a series of tests in the NIR band, the visible portion of the electromagnetic spectrum, and the UV, X-ray, and gamma radiation regions. The simple experiments pointed to interesting nanostructural properties previously theoretically assumed or modeled [14]-[16]. During the X-ray exposure, the anti-reflective effect was identified on the siliceous glass substrate, and we assume that such an event can be observed also with other configurations of the layers and substrate. In the gamma ray examination, the electromagnetic wave attenuation differed between the exposed sides, as predicted, and, additionally, we then detected an effect related to the resonant frequency of the nanomaterial structure.

The results are applicable in different sectors of the current research into the propagation and modeling of specific problems of the electromagnetic region.

\section{ACKNOWLEDGMENT}

The research was funded via a grant of the Czech Science Foundation (GA 17-00607S) and the National Sustainability Program, grant No. LO1401. For the actual analyses and experiments, infrastructure of the SIX Center was used; the X-ray measurement was ensured by the company Thermo Sanace s.r.o.

\section{REFERENCES}

[1] V. Chacko, S. Bansal, A.K. Hafiz. (2018). Effect of dispersion on omnidirectional reflection band in zinc oxide-based one-dimensional photonic crystal 
heterostructures. Journal of Nanophotonics, 12 (2), 026012. doi: 10.1117/1.JNP.12.026012.

[2] J. Wang, W. Lin, E. Cao, X. Xu, W. Liang, X. Zhang. (2017). Surface plasmon resonance sensors on raman and fluorescence spectroscopy. Sensors, 17 (12), 2719. doi:10.3390/s17122719.

[3] B. Zhou, Y. Dong, L. Liu, L. Chang, F. Bi, X. Wang. (2019). Enhanced soft magnetic properties of the Febased amorphous powder cores with novel $\mathrm{TiO}_{2}$ insulation coating layer. Journal of Magnetism and Magnetic Materials, 474, 1-8. doi: 10.1016/j.jmmm. 2018.11.014.

[4] A.A. Alkanoo, A. Sofyan, S.A. Taya. (2018). Theoretical investigation of five-layer waveguide structure including two left-handed material layers for refractometric applications. Journal of Magnetism and Magnetic Materials, 449, 395-400. doi: 10.1016/j. jmmm.2017.10.086.

[5] M. Meng, C.G. Shi, T. Li, S.E. Shi, T. Li, L.Z. Liu. (2017). Magnetism induced by cationic defect in monolayer $\mathrm{ReSe}_{2}$ controlled by strain engineering. Applied Surface Science, 425, 696-701. doi: 10.1016/ j.apsusc.2017.06.266

[6] J. Leng, Z. Zhou, F. Li, Q. Zheng, G. Liu. (2017). Speckle noise suppression using part of pixels in a single-exposure digital hologram. Optical Engineering, 56 (5), 053103. doi: 10.1117/1.OE.56.5.053103.

[7] M.T.H. Azar, M. Zavvari, A. Arashmehr, Y. Zehforoosh, P. Mohammadie. (2017). Design of a highperformance metal-insulator-metal plasmonic demultiplexer. Journal of Nanophotonics, 11 (2), 026002. doi: 10.1117/1.JNP.11.026002.

[8] M.F. Craciun, S. Russo, M. Yamamoto, S. Tarucha. (2011). Tuneable electronic properties in graphene. Nano Today, 6 (1), 42-60. doi: 10.1016/j.nantod.2010. 12.001 .

[9] C. Wang, R. Cheng, L. Liao, X. Duan (2013). High performance thin film electronics based on inorganic nanostructures and composites. Nano Today, 8 (5), 514530. doi: 10.1016/j.nantod.2013.08.001.

[10] K.J.A. Ooi, Y.S. Ang, J.L. Cheng, L.K. Ang, D.T.H. Tan. (2017). Electronic scattering of graphene plasmons, in the terahertz nonlinear regime. IEEE Journal of Selected Topics in Quantum Electronics, 23 (4). doi: 10.1109/JSTQE.2016.2615946.

[11] J.Y. Deng, K.F. Dong, Y.G. Peng, G. Ju, J. Hu, G.M. Chow, J.S. Chen. (2016). Effect of TiON-MgO intermediate layer on microstructure and magnetic properties of $\mathrm{L} 10 \mathrm{FePt}-\mathrm{C}-\mathrm{SiO} 2$ films. Journal of Magnetism and Magnetic Materials, 417, 203-207. doi: 10.1016/j.jmmm.2016.05.096.

[12] Y. Chen, H. Sun, W. Peng. (2017). 2D transition metal dichalcogenides and graphene-based ternary composites for photocatalytic hydrogen evolution and pollutants degradation. Nanomaterials, 7, 62. doi:10. 3390/nano7030062.

[13] P. Fiala, D. Nespor, P. Drexler, M. Steinbauer. (2016). Numerical model of a nanoelectric line from a graphene component. Microsystem Technologies, 22 (12), 27652782. doi: 10.1007/s00542-015-2772-4.

[14] P. Fiala, E. Gescheidtova, T. Jirku. (2009). Tuned structures for special $\mathrm{THz}$ applications. In Progress in Electromagnetics Research Symposium. Beijing, China, 151-155.

[15] R. Kadlec, P. Drexler. (2017). Analysing the responses of layered materials with varied parameters. In 2017 Progress in Electromagnetics Research Symposium Fall (PIERS - FALL). IEEE, 988-992. doi: 10.1109/ PIERS-FALL.2017.8293277.

[16] P. Drexler, D. Nespor, R. Kadlec, M. Cap. (2016). Numerical analysis of metallic periodic structures in $\mathrm{THz}$ region. In 2016 Progress in Electromagnetics Research Symposium (PIERS). IEEE, 2730-2733. doi: 10.1109/PIERS.2016.7735111.

[17] P. Fiala, P. Drexler, D. Nespor. (2014). Principal tests and verification of a resonance-based solar harvester utilizing micro/nano technology. Microsystem Technologies, 20 (4-5), 845-860. doi: 10.1007/s00542013-2063-x.

[18] R. Urban, P. Drexler, P. Fiala, D. Nespor. (2014). Numerical model of a large periodic structure. In Progress in Electromagnetics Research Symposium. Guangzhou, China, 2350-2354.

[19] P. Fiala, P. Drexler, D. Nespor. (2013). A resonancebased solar element: A numerical model and micro/nano technology application. In Smart Sensors, Actuators, and MEMS VI : Proceedings of SPIE 8763. doi: 10.1117/12.2015111.

[20] P. Fiala, P. Werner, P. Osmera, P. Dohnal. (2017). Using a multiscale toroidal element to model a hydrogen atom. In 2017 Progress in Electromagnetics Research Symposium - Fall (PIERS - FALL). IEEE, 956-960. doi: 10.1109/PIERS-FALL.2017.8293271.

[21] P. Fiala, P. Werner, P. Osmera, E. Gescheidtova, P. Drexler, T. Kriz. (2017). Periodical structures and multiscale modelling. In 2017 Progress in Electromagnetics Research Symposium - Spring (PIERS). IEEE, 1698-1703. doi: 10.1109/PIERS.2017. 8262022.

[22] P. Werner. (2018). Základy modelováni prstencové struktury elementárních částic hmoty. Brno, Czech Republic: Brno University of Technology, ISBN 97880-214-5620-4. (in Czech)

[23] J.A. Stratton. (1941). Electromagnetic Theory. Wiley.

[24] H. Kikuchi. (2001). Electrohydrodynamics in Dusty and Dirty Plasmas, Gravito-Electrodynamics and EHD. Kluwer.

[25] ANSYS. (1994-2019). Ansys Multiphysics Manuals. www.ansys.com.

[26] P. Drexler, R. Kadlec, K. Bartušek, P. Fiala, R. Kubásek. (2010). Magnetoinductive lens for experimental mid-field MR tomograph. In Progress in Electromagnetics Research Symposium Proceedings. Cambridge, UK, 1047-1050.

[27] D. Nespor, P. Drexler, R. Kadlec, E. Gescheidtova. (2016). Design and numerical modeling of metaldielectric resonant structures for infrared band. In 26th 
International Conference Radioelektronika. IEEE, 458462. doi: 10.1109/RADIOELEK.2016.7477405.

[28] F.P. Netzer, S. Surnev. (2010). STM imaging of oxide nanolayer model systems, scanning tunneling microscopy in surface science. In Scanning Tunneling Microscopy in Surface Science. Wiley, 147-188. doi: 10.1002/9783527628827.ch6.

[29] R. Kadlec, P. Fiala. (2015). The response of layered materials to EMG waves from a pulse source. Progress in Electromagnetics Research M, 42 (1), 179-187. doi: 10.2528/PIERM15042904.

[30] L.E. Vieira, K.C. Kleinjohanna, J.B.R. Neto, A.N. Klein, D. Hotza, R. Moreno. (2016). Dip coating of a carbon steel sheet with $\mathrm{Ni}$ reinforced $\mathrm{TiO}_{2}$ nanoparticles. Materials Research, 19 (3), 648-653. doi: 10.1590/1980-5373-MR-2016-0006.

[31] J.-M. Song, J.-S. Lee. (2016). Self-assembled nanostructured resistive switching memory devices fabricated by templated bottom-up growth. Nature: Scientific Reports, 6, 18967. doi: 10.1038/ srep18967.

[32] P.R. Wallace. (1947). The band theory of graphite. Physical Review, 71, 622-634. doi: 10.1103/PhysRev. 71.622.

[33] F. Qin, H. Xia, Y. Peng, Z. Wu. (2018). Integrated modeling, simulation, and visualization for nanomaterials. Complexity, 2018, 5083247. doi: 10.1155/2018/5083247

[34] F.J. Ferrández-Pastor, J.M. García-Chamizo, M. NietoHidalgo. (2017). Electromagnetic differential measuring method: Application in microstrip sensors developing. Sensors, 17, 1650. doi: 10.3390/ s17071650.

[35] G. Kang, J. Yoo, J. Ahn, K. Kim. (2015). Transparent dielectric nanostructures for efficient light management in optoelectronic applications. Nano Today, 10 (1), 2247. doi: 10.1016/j.nantod.2015.01.008.
[36] P. Xu, M.S. Gordon. (2014). Renormalized coupled cluster approaches in the cluster-in molecule framework: Predicting vertical electron binding energies of the anionic water clusters $\left(\mathrm{H}_{2} \mathrm{O}\right) \mathrm{n}(-)$. The Journal of Physical Chemistry A, 118 (35), 7548-7559. doi: 10.1021/jp5015498.

[37] A.R. Vázquez-Velázquez, M.A. Velasco-Soto, S.A. Pérez-García, L. Licea-Jiménez. (2018). Functionalization effect on polymer nanocomposite coatings based on $\mathrm{TiO} 2-\mathrm{SiO} 2$ nanoparticles with superhydrophilic properties. Nanomaterials, 8 (6), 369. doi:10.3390/nano8060369.

[38] S. Das, G.C. Nayak, S.K. Sahu, P.C. Routray, A.K. Roy, H. Baskey. (2015). Microwave absorption properties of double-layer composites using $\mathrm{CoZn} / \mathrm{NiZn} / \mathrm{MnZn}$-ferrite and titanium dioxide. Journal of Magnetism and Magnetic Materials, 377, 111-116. doi: 10.1016/ j.jmmm.2014.10.059.

[39] P. Uudeküll, J. Kozlova, H. Mändar, et al. (2017). Atomic layer deposition of titanium oxide films on Assynthesized magnetic Ni particles: Magnetic and safety properties. Journal of Magnetism and Magnetic Materials, 429, 299-304. doi: 10.1016/j.jmmm.2017. 01.045 .

[40] M. Platunov, N. Kazak, V. Dudnikov, et al. (2019). Element selective magnetism in $\mathrm{Ho} 0.5 \mathrm{Nd} 0.5 \mathrm{Fe} 3(\mathrm{BO} 3) 4$ single crystal probed with hard X-ray magnetic circular dichroism. Journal of Magnetism and Magnetic Materials, 479, 312-316. doi: 10.1016/j.jmmm.2019.02.040.

Received February 25, 2019 Accepted July 30, 2019 